\title{
Circumstellar envelopes of Cepheids: a possible bias affecting the distance scale?
}

\author{
Pierre Kervella, ${ }^{1}$ Alexandre Gallenne, ${ }^{2}$ and Antoine Mérand ${ }^{3}$ \\ ${ }^{1}$ LESIA, Observatoire de Paris, CNRS UMR 8109, UPMC, Université Paris Diderot, \\ 5 Place Jules Janssen, F-92195 Meudon, France \\ email: pierre.kervella@obspm.fr
}

${ }^{2}$ Universidad de Concepción, Departamento de Astronomía, Casilla 160-C, Concepción, Chile

${ }^{3}$ European Southern Observatory, Alonso de Córdova 3107, Casilla 19001, Santiago 19, Chile

\begin{abstract}
Circumstellar envelopes (CSEs) have been detected around many Cepheids, first based on long-baseline interferometry, and now also using other observing techniques. These envelopes are particularly interesting for two reasons: their presence could impact the Cepheid distance scale, and they may be valuable tracers of stellar mass loss. Here we focus on their potential impact on the calibration of the Cepheid distance scale. We consider the photometric contribution of the envelopes in the visible, near-, and thermal-infrared domains. We conclude that the impact of CSEs on the apparent luminosities of Cepheids is negligible at visible wavelengths and generally weak $(<5 \%)$ in the near-infrared $(\lambda \approx 2 \mu \mathrm{m})$. In the thermal-infrared domain $(\lambda \approx 8 \mu \mathrm{m})$, the flux contribution of the CSEs differs depending on the pulsation period: it is relatively weak $(<15 \%)$ for stars with periods shorter than $P \approx 10$ days, but can reach $\approx 30 \%$ for long-period Cepheids. We specifically discuss the long-period Galactic Cepheid RS Puppis, which exhibits a very large circumstellar, dusty envelope, and we conclude that this is not a representative case. Overall, the contribution of CSEs to the usual period-luminosity relations (from the visible to the $K$ band) is mostly negligible. They could affect calibrations at longer wavelengths, although the presence of envelopes may have been partially taken into account in the existing empirical calibrations.
\end{abstract}

Keywords. Cepheids, stars: circumstellar matter, stars: distances

\section{Introduction}

Cepheids are historically the most important class of variable stars, thanks to the relation between their pulsation period and absolute luminosity, which played a key role in the discovery of the expansion of the Universe. In particular, long-period Cepheids are so bright that they can be observed in very distant galaxies and act as standard candles to measure their distances. Although they remain fundamental distance indicators, the circumstellar envelopes (CSEs) recently discovered around several Galactic Cepheids may create a positive bias affecting their apparent luminosities. In this paper, we discuss the properties of these envelopes and their potential impact on the period-luminosity relation calibration in different wavelength domains (visible, near-infrared, thermal infrared).

\section{Envelope properties in the near- and thermal-infrared domains}

Near infrared $(\lambda \approx 2 \mu \mathrm{m})$ : Compact CSEs around Cepheids were initially discovered through interferometry in the near-infrared $K$ band $(\lambda \approx 2.2 \mu \mathrm{m})$. They were detected around the long-period Cepheid $\ell$ Car $(P=35.5$ days; Kervella et al. 2006), Polaris, $\delta$ Cep, and Y Oph (Mérand et al. 2006, 2007). The envelopes of $\ell$ Car and RS Pup have also been studied by Kervella et al. (2009), who determined their photometric properties, 
and a hydrogen-rich envelope was spatially resolved around RS Pup by Gallenne et al. (2009) using adaptive optics. The photometric contribution of the envelopes relative to the stellar flux is estimated at between 1.5 and 5\%. As suggested by Mérand et al. (2007), there is apparently a correlation between the CSE flux contribution and the period of the Cepheid, with longer periods exhibiting more dominant CSEs, but the number of stars is still too limited to reach firm conclusions. It is, however, interesting to note that CSEs have been found around all Cepheids observed using interferometry, which is an indication that the presence of envelopes around Cepheids may be ubiquitous.

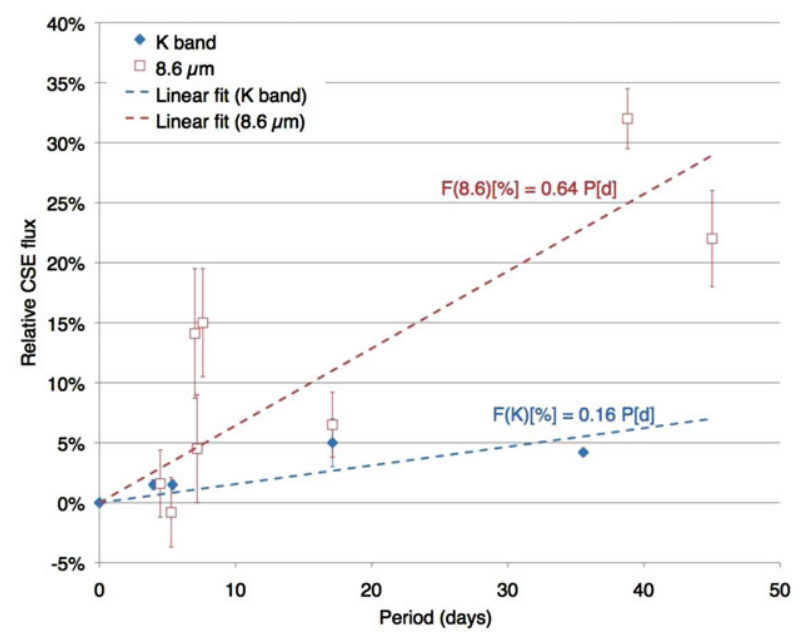

Figure 1. Relative flux contribution of Cepheid envelopes at $2 \mu \mathrm{m}$ ( $K$ band) and $8 \mu \mathrm{m}$ (data from Mérand et al. 2007; Gallenne et al. 2012).

Thermal infrared $(\lambda \gtrsim 8 \mu \mathrm{m})$ : Gallenne et al. (2012) obtained diffraction-limited imaging in the thermal-infrared domain $(8-12 \mu \mathrm{m})$ using VLT/VISIR. Of our sample of eight classical Cepheids, seven show a thermal-infrared excess characteristic of the presence of warm circumstellar material. The measured excess reaches more than $30 \%$ of the stellar flux for long-period Cepheids. For four of these stars, the envelopes appear spatially resolved on a scale of approximately $1^{\prime \prime}$. For RS Pup and $\ell$ Car, Kervella et al. (2009) also detected the compact CSEs using thermal-infrared interferometry with the VLTI/MIDI instrument. Using the Spitzer Space Telescope, Marengo et al. (2010a,b) and Barmby et al. (2011) identified extended envelopes around many Cepheids, in particular for $\delta$ Cep, which exhibits a spectacular bow shock at the interface of its stellar wind and the interstellar medium.

The relative flux of the CSE increases with the Cepheid's period, both at near- and thermal-infrared wavelengths, as shown in Fig. 1. This increase in CSE brightness, if caused by an increased mass-loss rate, is consistent with the theoretical predictions by Neilson \& Lester (2008), based on a pulsation-driven mass-loss scenario. As proposed by Neilson et al. (2011), such mass loss could also provide an explanation for the longstanding pulsational versus evolutionary mass discrepancy of Cepheids.

\section{The particular case of the long-period Cepheid RS Pup}

RS Pup appears to be anomalous, since it is the only known example of a Cepheid surrounded by a large dusty nebula (see Fig. 2, left). This nebula scatters the light from the star, creating spectacular light echoes (Kervella et al. 2008). Based on VLT/FORS 
polarimetric imaging, Kervella et al. (2012) estimated the mass of the scattering dust at $\approx 3 \mathrm{M}_{\odot}$ (see Fig. 2, right) and concluded that RS Pup could not create such a massive nebula through stellar mass loss. The location of RS Pup in exceptionnally dense interstellar medium provides a natural explanation of the scarcity of similar Cepheid-nebula associations. This also confirms that the presence of similar very large and cold dusty nebulae around Cepheids is probably very rare, and therefore not a significant source of photometric bias for the calibration of period-luminosity relations.

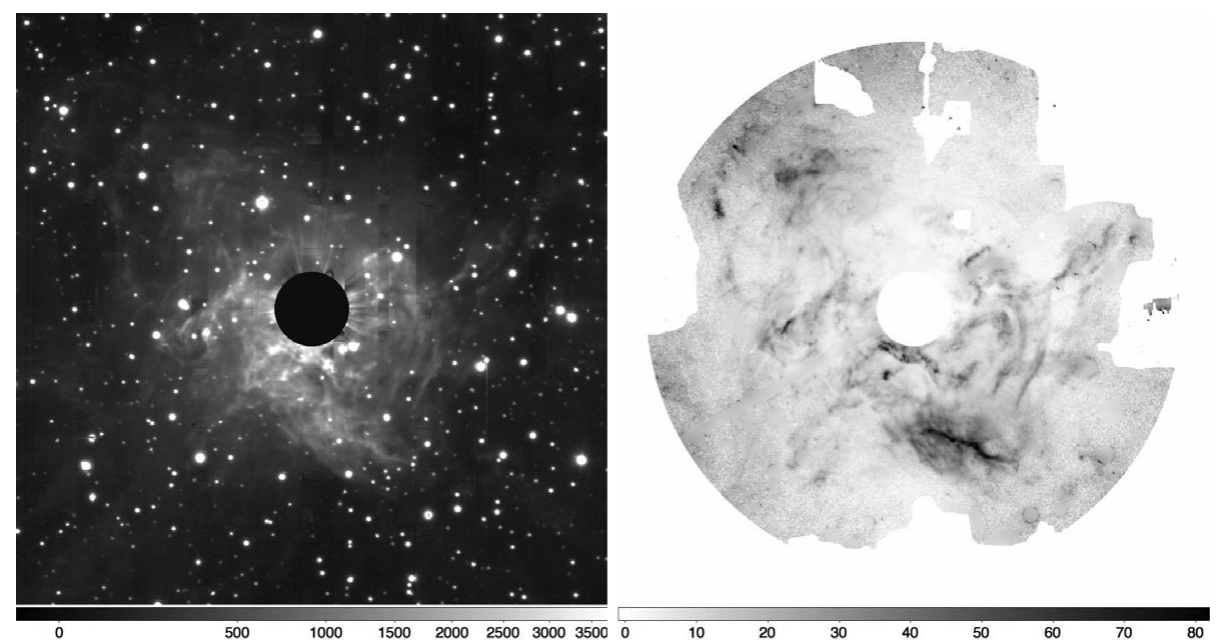

Figure 2. (left) Scattered-light image of the nebula surrounding RS Pup (in FORS counts). (right) Map of the dust density in the nebula (in $\mathrm{H} a \operatorname{arcsec}^{-2}$ ) derived by Kervella et al. (2012).

\section{Impact of envelopes on the apparent luminosity of Cepheids and period-luminosity relations}

The typical photometric contribution of Cepheid CSEs, as a function of wavelength and pulsation period is summarized in Table 1:

Visible: At visible wavelengths, the CSE luminosity is very small (probably $<1 \%$ ). Such a contribution is negligible for the calibration of period-luminosity relations, in particular compared to the uncertainties in the interstellar reddening correction.

Near-infrared: The current accuracy of the calibration of the Cepheid period-luminosity relations in the near-infrared domain is around 0.03 mag (see, e.g., Fouqué et al. 2007; Storm et al. 2011). As the expected contribution from the Cepheid CSEs is estimated at around $5 \%$, it is in most cases negligible. However, note that this photometric contribution is systematically positive, and may therefore create a deviation of the statistical properties of the Cepheid positions in the period-luminosity diagram.

Thermal infrared: In this wavelength domain, the CSE flux can reach a very significant fraction of the flux of the star, and introduce a strong bias in its apparent luminosity. It remains limited to $\approx 5 \%$ for short-period Cepheids $(P<10$ days $)$, but long-period Cepheids often exhibit considerable thermal-infrared excesses, as shown by Gallenne et al. (2012; see also Fig. 1). Recent period-luminosity calibrations between 3.5 and $8 \mu \mathrm{m}$ have typical uncertainties on the order of $0.05 \mathrm{mag}$ in the slope and zero point (see, e.g., Ngeow et al. 2010; Scowcroft et al. 2011). The impact of CSEs on these empirical calibrations at intermediate wavelengths is probably on the order of the statistical uncertainties, and it may have been taken into account in the derivation of the slopes of the relations. For 
Table 1. Photometric contribution of Cepheid envelopes, relative to the star's flux, as a function of pulsation period and wavelength.

\begin{tabular}{lccc}
\hline Period & Visible & $\lambda=2 \mu \mathrm{m}$ & $\lambda=8 \mu \mathrm{m}$ \\
\hline$<10$ days & negligible & $\lesssim 3 \%$ & $\lesssim 15 \%$ \\
10-30 days & negligible & $\lesssim 5 \%$ & $\lesssim 20 \%$ \\
$>30$ days & negligible & $\lesssim 5 \%$ & $\lesssim 35 \%$ \\
\hline
\end{tabular}

longer infrared wavelengths $(\lambda>8 \mu \mathrm{m})$, the CSE envelope contribution can be significant for all Cepheid periods.

\section{Conclusion}

The photometric contribution of Cepheid CSEs should be taken into account when using Cepheids as standard candles in the thermal-infrared domain $(\lambda \geqslant 8 \mu \mathrm{m})$. At shorter wavelengths, their effect on the apparent luminosity of Cepheids is usually small or negligible compared to other sources of uncertainty in the Cepheid distance scale (e.g., reddening corrections, statistical dispersion). Very large dusty envelopes such as that of RS Pup are probably very rare and are most probably not a significant source of bias for the distance scale.

\section{References}

Barmby, P., Marengo, M., Evans, N. R., et al. 2011, AJ, 141, 42

Fouqué P., Arriagada P., Storm J., et al. 2007, A\&A, 476, 73

Gallenne, A., Mérand, A., Kervella, P., \& Girard, J. H. V. 2009, A\&AA, 527, 51

Gallenne, A., Kervella, P., \& Mérand, A. 2012, A\&SA, 538, A24

Kervella, P., Mérand, A., Perrin, G., \& Coudé du Foresto, V. 2006, A\&̈A, 448, 623

Kervella, P., Mérand, A., Szabados L., et al. 2008, A\&A, 480, 167

Kervella, P., Mérand, A., \& Gallenne, A. 2009, A $\& A$, 498, 425

Kervella, P., Mérand, A., Szabados L., et al. 2012, A\&A, 541, A18

Marengo, M., Evans, N. R., Barmby, P., et al. 2010a, ApJ, 709, 120

Marengo, M., Evans, N. R., Barmby, P., et al. 2010b, ApJ, 725, 2392

Mérand, A., Kervella, P., Coudé du Foresto, V., et al. 2006, A\&3A, 453, 155

Mérand, A., Aufdenberg, J., Kervella, P., et al. 2007, ApJ, 664, 1093

Neilson, H. R. \& Lester, J. B. 2008, ApJ, 684, 569

Neilson, H. R., Cantiello, M., \& Langer, N. 2011, A\&A, 529, L9

Ngeow, C.-C., Ita, Y., Kanbur, S. M., et al. 2010, MNRAS, 408, 983

Scowcroft, V., Freedman, W. L., Madore, B. F., et al. 2011, ApJ, 743, 76

Storm, J., Gieren, W., Fouqué, P., et al. 2011, A\&BA, 534, A94 